\title{
THE WEAK RADICAL OF A RING
}

\author{
KWANGIL KOH AND A. C. MEWBORN
}

1. Introduction. In [2] a weakly transitive ring of linear transformations is defined as follows:

Definition A. Let $V$ be a left vector space over a division ring $D$ and let $R$ be a ring of linear transformations of $V$. Consider $V$ as right $R$-module. Then $R$ is weakly transitive provided there is a right order $K$ in $D$ and a $(K, R)$-submodule $M$ of $V$ such that $M$ is uniform as $R$-module, $D M=V$, and such that if $\left\{m_{i}\right\}_{i=1}^{n}$ is a finite $D$-linearly independent subset of $M$ and if $\left\{y_{i}\right\}_{i=1}^{n}$ is a sequence from $M$, then there exists $r \in R, k \in K, k \neq 0$, such that $m_{i} r=k y_{i}, 1 \leqq i \leqq n$.

By a weakly transitive representation of a ring $R$ we mean a homomorphism of $R$ onto a weakly transitive ring.

Definition 1.1. If $R$ is a ring the weak radical, $W(R)$, of $R$ is the intersection of the set of all ideals which are kernels of weakly transitive representations. If $R$ has no weakly transitive representation, then $W(R)=R$.

Of importance in studying weakly transitive rings is the notion of an almost maximal right ideal, which is also defined in [2].

Definition B. If $I$ is a proper right ideal of a ring $R$ then $I$ is almost maximal provided that

(1) if $J_{1}$ and $J_{2}$ are right ideals of $R$ and $J_{1} \cap J_{2}=I$, then $J_{1}=I$ or $J_{2}=I$, i.e. $I$ is irreducible,

(2) if $a \in R$ and $[I: a]^{1} \supset I$, then $a \in I$,

(3) if $J$ is a right ideal of $R, J \supset I$, then $N(I) \cap J \supset I$, where $N(I)=\{r \in R: r I \subseteq I\}$, and if $a \in R$ such that $[J: a] \supseteq I$ then $[J: a] \supset I$.

It was shown in [2] that if $I$ is an almost maximal right ideal of $R$, then $M=R / I$ is a uniform right $R$-module with centralizer $K=N(I) / I$. $M$ has extended centralizer $D$ [see 2, p. 67] in which $K$ is a right order, and $M$ can be extended to a $(D, R)$-module $V$ such that $D M=V$ and such that $R$ induces a weakly transitive ring of linear transformations of the $D$-space $V$.

In this paper we observe that $W(R / W(R))=(0)$ and prove that $W(R)$ is the intersection of the set of almost maximal right ideals of $R$. From the fact that a (two-sided) ideal of a weakly transitive ring is also weakly transitive we deduce that if $S$ is an ideal of $R$ then $W(S)=W(R) \cap S$. Finally, if $n$ is a positive integer then $W\left(R_{n}\right)=W(R)_{n}$.

Received by the editors June 20, 1966.

${ }^{1}$ By $[I: a]$, we mean the set $\{r \in R: a r \in I\}$. 
It is interesting to note that in severel instances the usual proofs of the analogues of our theorems in the Jacobson structure theory (See, for example, [1, p. 10]) make very strong use of the notion of quasi-regularity. Since in the weak radical theory we have no analogue of this notion the proofs which we give suggest proofs of the classical theorems which do not depend on quasi-regularity. The authors have been unable to devise a proof of the equality of the left and right radicals without using quasi-regularity and this problem for the weak radical remains open.

From the definition of $W(R)$ it follows that if $W(R)=(0)$, then $R$ is a subdirect sum of weakly transitive rings. It is likely that this hypothesis of "weak semisimplicity" will serve in many cases where a ring is assumed to be semisimple.

2. The proof of the following theorem is straightforward and will be omitted.

THEOREM 2.1. If $R$ is a ring then $W(R / W(R))=(0)$.

Lemma 2.2. If $I$ is an almost maximal right ideal of a ring $R$ and $M=R / I$, then for each $m \in M, m \neq 0$, the right ideal $\mathrm{m}^{\gamma}=\{r \in R: m r=0\}$ is almost maximal.

Proof. $m R$ is a submodule of $M$ and hence is uniform. Since $m R \cong R / m^{\gamma}, m^{\gamma}$ is an irreducible right ideal. Suppose $a \in R$ and $\left[m^{\gamma}: a\right]=(m a)^{\gamma} \supset m^{\gamma}$. By Lemma 3.5 of $[2]$ it follows that $m a=0$; i.e. $a \in m^{\gamma}$.

Now suppose $J$ is a right ideal of $R$ and $J \supset m^{\gamma} . m J \neq(0)$, so there exists $j \in J$ with $m j \neq 0$. It follows directly from the proof of Theorem 3.7 of [2] that there exists $b \in R$ such that $(m j b)^{\gamma}=m^{\gamma}$ (in the terminology of [2] $m j b$ and $m$ are $D$-linearly dependent and $m j b \neq 0$ ). Then $j b \in J \cap N\left(m^{\gamma}\right)$ and $j b \notin m^{\gamma}$. Hence $J \cap N\left(m^{\gamma}\right) \supset m^{\gamma}$.

Again assume $J \supset m^{\gamma}$, and let $r \in R$ such that $[J: r] \supseteq m^{\gamma}$. We show $[J: r] \supset m^{\gamma}$. First suppose $(m r)^{\gamma}=m^{\gamma}$. Then $m J \neq(0)$ and $r J+m^{\gamma} \supset m^{\gamma}$. Let $n \in\left(r J+m^{\gamma}\right) \cap J, n \notin m^{\gamma}$. We can choose $n=r j, j \in J$. Then $n \in J$ implies $j \in[J: r]$. Also $r j=n \notin m^{\gamma}$ implies $j \notin(m r)^{\gamma}=m^{\gamma}$. Hence $[J: r] \supset m^{\gamma}$. Now suppose $(m r)^{\gamma} \neq m^{\gamma}$. Again by Lemma 3.5 of [2] there exists $a \in R$ such that $m r a=0$ and $m a \neq 0$. Then $a \in\left[m^{r}: r\right]$ $\subseteq[J: r]$ but $a \notin m^{\gamma}$. Hence $[J: r] \supset m^{\gamma}$.

The lemma is proved.

TheOREM 2.3. If $W(R) \neq R$, then $W(R)$ is the intersection of the set of almost maximal right ideals of $R$.

Proof. Let $I$ be an almost maximal right ideal and let $K$ be the 
kernel of the associated weakly transitive representation of $R$, i.e. $K=\{r \in R: R r \subseteq I\}$. If $n \in N(I), n \in I$, then $[I: n]=I$. It follows that $K \subseteq[I: n]=I$. Therefore $W(R)$ is contained in the intersection of the set of almost maximal right ideals.

Conversely, if $r$ is in the given intersection, then for any almost maximal right ideal $I$ we have $r \in \cap\left\{m^{\gamma}: m \in R / I\right\}$ by the lemma. This implies that $r$ is in the kernel of each representation associated with an almost maximal right ideal. Hence $r \in W(R)$. The theorem is proved.

THEOREM 2.4. If $R$ is a ring and $\phi$ is a homomorphism of $R$, then $W(R) \phi \subseteq W(R \phi)$.

Proof. Suppose $\psi$ is a weakly transitive representation of $R \phi$. Then $\phi \psi$ is a weakly transitive representation of $R$. Hence $W(R) \phi \subseteq \operatorname{Ker} \psi$.

TheOREM 2.5. Suppose $R$ is a weakly transitive ring of linear transformations and $J$ is a (two-sided) ideal of $R$. Then $J$ is also weakly transitive.

Proof. Suppose $R$ acts in the (left) vector space $V$ over a division ring $D$. Let $K$ and $M$ be as given in Definition A. Clearly $M$ is a $(K, J)$-submodule of $V$ and $M$ is uniform as $J$-module. Suppose $\left\{m_{i}\right\}_{i=1}^{n}$ is a finite $D$-linearly independent subset of $M$ and $\left\{y_{i}\right\}_{i=1}^{n}$ is a sequence from $M$. Let $a \in J, a \neq 0$, and let $m \in M$ such that $m a \neq 0$. There exist $\left\{r_{j}\right\}_{j=1}^{n}$ in $R$ and $\left\{k_{j}\right\}_{j=1}^{n}$ in $k, k_{j} \neq 0$, such that $m_{i} r_{j}=0, j \neq i$ and $m_{i} r_{i}=k_{i} m$. Let $I$ be an almost maximal right ideal such that $M=R / I$. Since $m_{i} r_{i} a=k_{i} m a \neq 0$, each $i$, there exist $\left\{s_{i}\right\}_{i=1}^{n}$ in $R, s_{i} \in I$ such that $m_{i} r_{i} a s_{i}=k_{i}^{\prime} m_{i}$, where $k_{i}^{\prime} \in K, k_{i}^{\prime} \neq 0$, $1 \leqq i \leqq n$. Now $\left\{k_{i}^{\prime} m_{i}\right\}_{i=1}^{n}$ is a $D$-linearly independent subset of $M$. Hence there exists $r^{\prime} \in R, k \in K, k \neq 0$, such that $k_{i}^{\prime} m_{i} r^{\prime}=k y_{i}, 1 \leqq i \leqq n$. Let $r=\sum_{i=1}^{n} r_{i} a s_{i} r^{\prime}$. Then $r \in J$ and $m_{i} r=k y_{i}, 1 \leqq i \leqq n$. Therefore $J$ is weakly transitive.

Theorem 2.6. If $R$ is a ring and $S$ is a two-sided ideal of $R$, then $W(S)=W(R) \cap S$.

Proof. Let $S \neq(0)$. Suppose $a \in S, a \in W(R)$. Let $\phi$ be a weakly transitive representation of $R$ such that $\phi(a) \neq 0 . \phi(R)$ is a weakly transitive ring and $\phi(S)$ is a nonzero two-sided ideal of $\phi(R)$. By Theorem $2.5, \phi(S)$ is a weakly transitive ring. Hence $a \notin W(S)$. Therefore $W(S) \subseteq W(R) \cap S$.

Conversely, suppose $a \in S, a \in W(S)$. Let $I$ be an almost maximal right ideal of $S$ such that $S a \nsubseteq I$. Let $M=S / I$ and let $m \in M$ 
such that $m a \neq 0$. There exists $s_{0} \in S$ such that $m^{\gamma}=\{s \in S: m s=0\}$ $=\left\{s \in S: m s_{0} s=0\right\}=\left(m s_{0}\right)^{\gamma}$. Then $m s_{0} a \neq 0$. Let $I^{*}=\left\{r \in R: m\left(s_{0} r\right)\right.$ $=0\}$. $I^{*}$ is clearly closed under addition. Suppose $r \in I^{*}, t \in R$. If $r t \notin I^{*}$ then $m\left(s_{0} r t\right) \neq 0$. Hence there exists $s \in S$ such that $0 \neq m\left(s_{0} r t\right) s$ $=m\left(s_{0} r\right)(t s)$, which is absurd. Thus $I^{*}$ is a right ideal of $R$. We show that $I^{*}$ is almost maximal in $R$.

Let $J^{*}$ be a right ideal of $R$ such that $J^{*} \supset I^{*}$. Let $j \in J^{*}, j \notin I^{*}$. Then $m\left(s_{0} j\right) \neq 0$. Hence there exists $s \in S$ such that $m\left(s_{0} j s\right) \neq 0$.Then $j s \in S \cap J^{*}, j s \notin I^{*}$. Thus $S \cap J^{*} \supset S \cap I^{*}=m^{\gamma}$. Since $m^{\gamma}$ is irreducible in $S$, it follows immediately that $I^{*}$ is irreducible in $R$.

Let $n \in N\left(m^{\gamma}\right)$ and $r \in I^{*}$. Suppose $m\left(s_{0} n r\right) \neq 0$. Then there exists $s \in S$ such that $m\left(s_{0} n r s\right) \neq 0$. Then $m(n r s) \neq 0$. Since $n \in N\left(m^{\gamma}\right)$, $r s \notin m^{\gamma}$. But $m\left(s_{0} r s\right)=m\left(s_{0} r\right) s=0$, which implies $r s \in\left(m s_{0}\right)^{\gamma}=m^{\gamma}$, a contradiction. Therefore $N\left(m^{\gamma}\right) \subseteq N\left(I^{*}\right)$. Also $\left(S \cap J^{*}\right) \cap N\left(m^{\gamma}\right) \supset m^{\gamma}$. If $t \in S \cap J^{*} \cap N\left(m^{\gamma}\right), t \notin m^{\gamma}$, then $m s_{0} t \neq 0$. Hence $t \notin I^{*}$, but $t \in J^{*} \cap N\left(I^{*}\right)$. Thus $J^{*} \cap N\left(I^{*}\right) \supset I^{*}$.

Now let $J^{*} \supset I^{*}$ and let $r \in R$ such that $r J^{*} \subseteq I^{*}$. If $r \notin I^{*}$, then $m\left(s_{0} r\right) \neq 0$. Since $J^{*} \cap S \supset m^{\gamma}$, we cannot have $\left[m\left(s_{0} r\right)\right]^{r} \supseteq J^{*} \cap S$. Hence there exists $j \in J^{*} \cap S$ such that $m\left(s_{0} r j\right) \neq 0$. Then $r j \in I^{*}$, a contradiction. Hence $r \in I^{*}$.

Again suppose $J^{*} \supset I^{*}$ and $r \in R$ such that $\left[J^{*}: r\right] \supseteq I^{*}$. We show $\left[J^{*}: r\right] \supset I^{*}$. Suppose $\left[J^{*}: r\right]=I^{*}$. Let $J=J^{*} \cap S \supset m^{\gamma}$. Assume first that $\left[m\left(s_{0} r\right)\right] r=m^{\gamma}$. Then $m\left(s_{0} r J\right) \neq(0)$. Hence $r J \Phi\left(m s_{0}\right)^{\gamma}=m^{\gamma}$, and $r J+m^{\gamma} \supset m^{\gamma}$. Then there exists $n \in\left(r J+m^{\gamma}\right) \cap J, n \in m^{\gamma}$. We can choose $n=r j, j \in J$. If $s \in m^{\gamma}$ then $s \in\left[m\left(s_{0} r\right)\right]^{\gamma}$, and $m\left(s_{0} r s\right)=0$. Then $r s \in\left(m s_{0}\right)^{\gamma}=m^{\gamma}$. Hence $r\left(m^{\gamma}\right) \subseteq m^{\gamma}$. Since $r j \notin m^{\gamma}, j \notin m^{\gamma}$. But $j \in\left[J^{*}: r\right]$. Hence $\left[J^{*}: r\right] \supset I^{*}$. Now assume that $\left[m\left(s_{0} r\right)\right]^{\gamma} \neq m^{r}$, then $\left[m\left(s_{0} r\right)\right]^{r} \Phi m^{\gamma}$, so there exists $a \in S$ such that $m s_{0} r a=0$ but $m a \neq 0$. Then $r a \in I^{*} \subseteq J^{*}$ implies $a \in\left[J^{*}: r\right]$. But $a \notin m^{\gamma}=\left(m s_{0}\right)^{\gamma}$ so $a \notin I^{*}$. Thus $\left[J^{*}: r\right] \supset I^{*}$.

It follows that $I^{*}$ is almost maximal in $R$. Since $a \notin I^{*}$, it follows from Theorem 2.3 that $a \notin W(R)$. Therefore $W(R) \cap S \subseteq W(S)$.

TheOREM 2.7. If $R$ is a ring and $n$ is a positive integer, then $W\left(R_{n}\right)=W(R)_{n}$.

Proof. $W\left(R_{n}\right) \subseteq W(R)_{n}$. Let $\alpha=\left(a_{i j}\right) \in R_{n}, \alpha \notin W(R)_{n}$. Assume $a_{k l} \notin W(R)$. By Theorem 2.3 there is an almost maximal right ideal $I$ of $R$ such that $a_{k l} \notin I$. Let $I^{*}=\left\{\left(b_{i j}\right) \in R_{n}: b_{k j} \in I, 1 \leqq j \leqq n\right\}$. We show that $I^{*}$ is an almost maximal right ideal of $R_{n}$. Since $\alpha \notin I^{*}$ it will follow by 2.3 that $\alpha \notin W\left(R_{n}\right)$. For simplicity of notation we give the proof for $k=1$.

Assume $J^{*}$ is a right ideal of $R_{n}$ and $J^{*} \supset I^{*}$. Let $\beta=\left(b_{i j}\right) \in J^{*}$, 
$\beta \notin I^{*}$. Suppose $b_{1 s} \notin I$. Then there exists $b \in R$ such that $b_{1 s} b \in N(I)$, $b_{1 s} b \in I$. Let $\gamma=\left(c_{i j}\right)$, where $c_{s 1}=b$ and $c_{i j}=0, i \neq s$ or $j \neq 1$. Then $\beta \gamma=\rho=\left(r_{i j}\right)$, where $r_{11}=b_{1 s} b$ and $r_{1 j}=0, j>1, \rho \in N\left(I^{*}\right), \rho \in I^{*}$. Hence $J^{*} \cap N\left(I^{*}\right) \supset I^{*}$. It is now clear that if $J_{1}^{*}$ and $J_{2}^{*}$ are right ideals of $R_{n}$ and $J_{1}^{*} \supset I^{*}, J_{2}^{*} \supset I^{*}$, then $J_{1}^{*} \cap J_{2}^{*} \supset I^{*}$.

Again assume that $J^{*} \supset I^{*}$ and let $\beta=\left(b_{i j}\right) \in R_{n}, \beta \notin I^{*}$. We show $\beta J^{*} \nsubseteq I^{*}$. Assume $b_{18} \notin I$. Clearly, if $s>1$. then $\beta J^{*} \Phi I^{*}$. Suppose $s=1$. Let $J=\left\{r \in R\right.$ : there exists $\left(c_{i j}\right) \in J^{*}$ with $\left.c_{11}=r\right\}$. Then $J \supset I$. So there exists $r \in J$ such that $b_{11} r \in I$. Choose $\gamma=\left(c_{i j}\right) \in J^{*}$ such that $c_{11}=r, c_{i j}=0$ for $i>1$. Then $\beta \gamma \notin I^{*}$.

Let $J^{*} \supset I^{*}$ and suppose $\beta=\left(b_{i j}\right) \in R_{n}$ such that $\beta I^{*} \subseteq J^{*}$. If $b_{11} \in I$, let $\gamma=\left(c_{i j}\right) \in R_{n}, \gamma \in I^{*}$, such that $c_{i j}=0$ for $i>1$. Then $\beta \gamma \in I^{*} \subset J^{*}$ implies $\left[J^{*}: \beta\right] \supset I^{*}$. Suppose $b_{11} \notin I$. Let $J=\{r \in R$ : there exists $\left(c_{i j}\right) \in J^{*}$ with $c_{11}=r, c_{i j}=0$ for $\left.j>1\right\} . J$ is a right ideal of $R$ and $J \supset I$, where the inclusion is proper because, as shown above, there is an element $\left(r_{i j}\right) \in J^{*} \cap N\left(I^{*}\right),\left(r_{i j}\right) \notin I^{*}$, such that $r_{i j}=0$ for $j>1$. Also $b_{11} I \subseteq J$. Hence $\left[J: b_{11}\right] \supset I$, because $I$ is almost maximal. Let $c \in\left[J: b_{11}\right], c \in I$. Choose $\gamma=\left(c_{i j}\right) \in R_{n}$ such that $c_{11}=c, c_{i j}=0$ for $i \neq 1$ or $j \neq 1$. Then $\beta \gamma \in J^{*}$ but $\gamma \in I^{*}$. Hence again $\left[J^{*}: \beta\right] \supset I^{*}$. It now follows that $I^{*}$ is an almost maximal right ideal of $R_{n}$

$W(R)_{n} \subseteq W\left(R_{n}\right)$. It is sufficient to show that any matrix in $W(R)_{n}$ with only one nonzero row must be in $W\left(R_{n}\right)$. For ease of notation we give the proof in the case where the nonzero row is the first row. Hence suppose $\alpha=\left(a_{i j}\right) \in R_{n}, \alpha \in W\left(R_{n}\right)$, and $a_{i j}=0$ for $i>1$. We show $\alpha \notin W(R)_{n}$.

There is an almost maximal right ideal $J^{*}$ of $R_{n}$ such that $\alpha \notin J^{*}$. There exists $\beta=\left(b_{i j}\right) \in N\left(J^{*}\right) \cap \alpha R_{n}, \beta \notin J^{*}$. If we show $\beta \in W(R)_{n}$ it will follow that $\alpha \notin W(R)_{n}$. Let $\gamma=\left(c_{i j}\right)$ be such that $c_{11}=b_{11}, c_{i j}=0$ for $i \neq 1$ or $j \neq 1$. Then $\gamma \beta=\beta^{2}$. $\beta^{2} \in J^{*}$ because $N\left(J^{*}\right) / J^{*}$ is an in tegral domain. Hence $\gamma \in J^{*}$.

Let $I^{*}=\left\{\rho \in R_{n}: \gamma \rho \in J^{*}\right\}$. By Lemma $2.2, I^{*}$ is an almost maximal right ideal of $R_{n}$. If $\rho \in I^{*}$, then $\gamma \rho \in J^{*}$. Since $\beta \in N\left(J^{*}\right), \beta \gamma \rho \in J^{*}$. But $\beta \gamma \rho=\gamma^{2} \rho$, hence $\gamma \rho \in I^{*}$. Thus $\gamma \in N\left(I^{*}\right), \gamma \in I^{*}$.

Let $I=\left\{r \in R\right.$ : there exists $\left(r_{i j}\right) \in I^{*}$ with $\left.r_{11}=r\right\}$. Then $I$ is a right ideal of $R$. Now $b_{11} \in I$; for suppose $\rho=\left(r_{i j}\right) \in I^{*}$ with $r_{11}=b_{11}$. We can assume $r_{i j}=0$ for $i>1$. Then $\gamma^{2}=\rho \gamma \in I^{*}$, which is impossible because $\gamma \in N\left(I^{*}\right), \gamma \in I^{*}$. We now show that $I$ is an almost maximal right ideal of $R$, from which it will follow that $b_{11} \notin W(R)$, and the theorem will be proved.

Suppose $J$ is a right ideal of $R$ and $J \supset I$. Let $r \in J, r \in I$. Define $\rho=\left(r_{i j}\right)$ with $r_{11}=r, r_{i j}=0$ for $i \neq 1$ or $j \neq 1$. Then $\rho \notin I^{*}$. Hence there exists $\tau=\left(t_{i j}\right) \in R_{n}$ such that $\rho \tau \in N\left(I^{*}\right)$ but $\rho \tau \notin I^{*}$. Then $r t_{11} \in N(I)$. 
But $r t_{11} \notin I$. For suppose $\mu=\left(m_{i j}\right) \in I^{*}$ with $m_{11}=r t_{11}$. Again we can suppose $m_{i j}=0$ for $i>1$. Then $\rho \tau \gamma=\mu \gamma$, which is a contradiction because $\rho \tau \gamma \notin I^{*}$ while $\mu \gamma \in I^{*}$. Thus $r t_{11} \in J \cap N(I), \quad r t_{11} \in I$, and $J \cap N(I) \supset I$.

We show now that $I^{*}=\left\{\left(r_{i j}\right): \quad r_{1 j} \in I, 1 \leqq j \leqq n\right\}$. Suppose $\rho=\left(r_{i j}\right) \in I^{*}$. Then clearly $r_{1 j} r \in I$ for each $r \in R, 1 \leqq j \leqq n$. But this implies, by the above argument, that $r_{1 j} \in I$. Conversely, suppose $r_{1 j} \in I, 1 \leqq j \leqq n$. If $\rho=\left(r_{i j}\right) \notin I^{*}$, then there exists $\tau \in R_{n}$ such that $\rho \tau \in N\left(I^{*}\right), \rho \tau \notin I^{*}$. Let $\rho \tau=\sigma=\left(s_{i j}\right)$. Again we may assume $s_{i j}=0$ for $i>1$. By the argument above, $s_{11} \in I$, a contradiction.

Assume $J_{1}$ and $J_{2}$ are right ideals of $R$ and $J_{1} \supset I, J_{2} \supset I$. Let $J_{\boldsymbol{k}}^{*}=\left\{\left(r_{i j}\right): r_{1 j} \in J_{k}, 1 \leqq j \leqq n\right\}, k=1,2$. Then $J_{1}^{*}$ and $J_{2}^{*}$ are right ideals of $R_{n}$ and $J_{k}^{*} \supset I^{*}, k=1,2$. Hence $J_{1}^{*} \cap J_{2}^{*} \supset I^{*}$, from which it follows easily that $J_{1} \cap J_{2} \supset I$.

Also, if $J \supset I$ and $r \in R$ such that $r I \subseteq J$, let $\sigma=\left(s_{i j}\right)$ with $s_{11}=r$, and $s_{i j}=0$ for $i \neq 1, j \neq 1$. Let $J^{*}=\left\{\left(r_{i j}\right): r_{1 j} \in J, 1 \leqq j \leqq n\right\}$. Then $\sigma I^{*} \subseteq J^{*}$. Hence $\left[J^{*}: \sigma\right] \supset I^{*}$, which clearly implies $[J: r] \supset I$. The theorem is proved.

\section{REFERENCES}

1. N. Jacobson, Structure of rings, Colloq. Publ., Vol. 37, Amer. Math. Soc., Providence, R. I., 1956.

2. K. Koh and A. C. Mewborn, A class of prime rings, Canad. Math. Bull. (1) 9 (1966), 63-72.

North Carolina State University at Raleigh and

The University of North Carolina at Chapel Hill 